\title{
Bladder cancer-associated protein is suppressed in human cervical tumors
}

 \\ ${ }^{1}$ Oncology Center, Renmin Hospital of Wuhan University; ${ }^{2}$ Wuhan Institute of Biologic Products; \\ ${ }^{3}$ Institute of Virology, School of Medicine, State Key Laboratory of Virology, \\ Wuhan University, Wuhan, Hubei, P.R. China
}

Received September 27, 2011; Accepted November 29, 2011

DOI: $10.3892 /$ etm.2011.408

\begin{abstract}
Bladder cancer-associated protein (BLCAP) is downregulated in bladder cancer and has been identified as a prognostic biomarker for human bladder cancer. We previously reported that BLCAP mRNA is decreased in cervical cancer tissues, and overexpression of BLCAP was found to inhibit cell growth and induce apoptosis in the human cervical cancer HeLa cell line To investigate the BLCAP protein expression in cervical cancer and its potential clinical indications, we developed a polyclonal antibody against human BLCAP to assess the BLCAP protein expression in 30 cervical cancer tissues and 30 non-tumor cervical tissues from patients. Western blotting data showed that a single band of recombinant protein was probed by antiserum of BLCAP and no band was probed by pre-immune serum. BLCAP expression was significantly downregulated in cervical carcinoma tissues compared with its expression in the non-tumor cervical tissues. Moreover, cervical carcinoma tissues from patients with stage III-IV had significantly lower BLCAP expression percentage compared with stage I-II. Similarly, a significantly lower BLCAP expression percentage was observed in moderately/poorly differentiated tumor tissues and in the tumor tissues from patients with lymphatic metastasis (LM) compared with welldifferentiated tumor tissues and non-LM patients, respectively. Our results suggest that decreased BLCAP protein expression is associated with poor prognosis and it could be a potential bio-index to predict cervical tumor patient outcome.
\end{abstract}

\section{Introduction}

Cervical carcinoma is the second leading cause of cancerrelated death in women worldwide, with over 500,000 new cases reported each year in developing countries. High-risk human papillomavirus (HPV) infection such as HPV16 and/or

Correspondence to: Professor Xinxing Wu, Institute of Virology, School of Medicine, Wuhan University, Wuhan, Hubei 430071, P.R. China

E-mail: wuxinxing9755@hotmail.com

Key words: bladder cancer-associated protein, cervical carcinoma
HPV18 is regarded as the major etiological factor for cervical carcinoma $(1,2)$, however, genetic factors may play an important role in its occurrence. The activation of oncogenes and/or the inactivation of tumor-suppressor genes play an important role in tumor development and progression $(3,4)$. Therefore, it is important to study the genes associated with cervical carcinoma at the molecular level.

The bladder cancer-associated protein $(B L C A P)$ gene (GenBank accession no. NM006698) is located on chromosome 20 and is a novel tumor-suppression gene identified from human bladder carcinoma. The mRNA level of BLCAP has been found to be markedly downregulated in bladder carcinoma $(5,6)$, as well as human tongue carcinoma (7). In our previous study, BLCAP mRNA expression was decreased in cervical tumor tissues (7) and overexpression of BLCAP was found to inhibit cell growth and to induce apoptosis in the human cervical cancer HeLa cell line (8). Since BLCAP may be a cervical carcinoma-related suppressor gene, it is important to determine protein expression levels in cervical carcinoma.

Here, we established a pET prokaryotic expression system to express the His-tagged BLCAP fusion protein to immune rabbits for preparing the polyclonal antibody. This purified BLCAP polyclonal antibody was used to detect the BLCAP protein expression level in 30 cervical carcinoma tissues and 30 normal cervical tissues.

\section{Materials and methods}

Plasmid construction. The primer sequences of the BLCAP were designed with Primer 5.0 software, and the coding region of the mature BLCAP protein was amplified using polymerase chain reaction (PCR). Primer 1 (5'-GCA GAA TTC ATG TAT TGC CTC CAG TG-3') and primer 2 (5'-GC AAG CTT TTA GGT GCC CAC AAC G-3') were synthesized by Invitrogen (Shanghai, China). Primer 1 was synthesized with an EcoRI site (shown in bold) and primer 2 was synthesized with a HindIII site (shown in bold). The amplification profile included one initial hot-start denaturing step at $94^{\circ} \mathrm{C}$ for $5 \mathrm{~min}$, followed by 30 cycles of the following conditions: $94^{\circ} \mathrm{C}$ for $1 \mathrm{~min}, 58^{\circ} \mathrm{C}$ for $30 \mathrm{sec}$, and $72^{\circ} \mathrm{C}$ for $1 \mathrm{~min}$, and a final extension at $72^{\circ} \mathrm{C}$ for $10 \mathrm{~min}$. The expression vector pET-32(a) was digested with EcoRI and HindIII. The digested pET-32(a) was purified by agarose gel and extracted using the QIAquick Gel Extraction kit. 
A recombinant plasmid was constructed by inserting the PCR amplified fragment (also digested with EcoRI and HindIII) into the pET-32(a) vector and transformed into the E. coli strain Rosetta. The transformants [pET-32(a)-BLCAP] were confirmed by PCR, restriction enzyme digestion and DNA sequencing.

Prokaryotic expression and purification of full length BLCAP. The prokaryotic expression vector pET-32a-BLCAP was introduced into the bacterial host $E$. coli strain Rosetta following standard protocol. Rosetta, the derivational strain of BL21, contains the extra gene copies for coding rare tRNA, which facilitates the prokaryotic expression of eukaryotic protein in $E$. coli Rosetta. The transformants were cultured in LB medium containing ampicillin $(100 \mu \mathrm{g} / \mathrm{ml})$ at $37^{\circ} \mathrm{C}$ in a shaking incubator until an OD600 of 0.8 to 1.0 was attained. Recombinant BLCAP expression was induced by adding $1 \mathrm{mM}$ isopropyl1-thio-D-galactopyranoside (IPTG) and further incubation at $37^{\circ} \mathrm{C}$ for $6 \mathrm{~h}$. The cells were harvested by centrifugation at $5,000 \mathrm{x} \mathrm{g}$ for $15 \mathrm{~min}$ at $4^{\circ} \mathrm{C}$, and the pellets were re-suspended in lysis buffer [50 mM Tris (pH 8.9), $100 \mathrm{mM} \mathrm{NaCl}, 1 \mathrm{mM}$ EDTA, $100 \mu \mathrm{g} / \mathrm{ml}$ lysozyme, $100 \mu \mathrm{g} / \mathrm{ml}$ phenylmethylsulfonyl fluoride (PMSF) and $1 \mu \mathrm{g} / \mathrm{ml}$ each of pepstatin, leupeptin and aprotinin], and further incubated at $4^{\circ} \mathrm{C}$ for $1 \mathrm{~h}$. Thereafter, the cells were sonicated and centrifuged at $12,000 \mathrm{x}$ g for $30 \mathrm{~min}$. The clear supernatant was collected and used for protein purification.

The recombinant protein was purified based on its $\mathrm{N}$-terminal $\mathrm{His}_{6}$-tag by affinity chromatography using a $\mathrm{Ni}^{2+}$-NTA HiTrap chelating Sepharose column (Qiagen) that was equilibrated with $20 \mathrm{ml}$ buffer A containing $10 \mathrm{mM}$ imidazole. The cell lysate was applied to the column and allowed to bind using a flow rate of $1.0 \mathrm{ml} / \mathrm{min}$. The bound protein was eluted by applying a gradient of $10-500 \mathrm{mM}$ imidazole in buffer A using a flow rate of $1.5 \mathrm{ml} / \mathrm{min}$. Peak fractions were collected between 200 and $300 \mathrm{mM}$ imidazole. The Bradford method was used to determine the protein concentration, and the protein was confirmed by western blot analysis using an anti-His monoclonal antibody.

Production and purification of polyclonal antibody against $B L C A P$. New Zealand white rabbits received an intradermal injection of BLCAP protein $(500 \mu \mathrm{g} / \mathrm{rabbit})$ mixed with CFA in a 1:1 ratio. After 4 week, the rabbits were boosted subsequently 3 times at two-week intervals with the BLCAP protein $(250 \mu \mathrm{g} / \mathrm{rabbit})$ mixed with IFA in a $1: 1$ ratio. Prior to immunization, blood samples were taken from the marginal vein of the rabbit ear, and the sera were obtained to determine the antibody titer by western blotting. The polyclonal antibody was purified by following a standard protocol for the purification of the antibody.

Western blot analysis and agar gel precipitin test. Purified proteins were analyzed by western blot analysis (12\% SDS PAGE gel) following a standard procedure. After an overnight blocking in 5\% BSA the membrane was incubated with an anti-BLCAP fusion protein polyclonal antibody for $1 \mathrm{~h}$ at room temperature (RT) and then incubated in alkaline phosphatase (Ap)-conjugated goat anti-rabbit IgG at a dilution of 1:1500 for $1 \mathrm{~h}$ at RT. The membrane was washed and the specific protein bands were visualized using 3,3'-diaminobenzidine
(DAB). To determine antibody titer, the agar gel precipitin test was performed as described previously with modification (9). Briefly, a cluster of six wells surrounding a center well was cut into the solidified agar. Antiserum $(25 \mu \mathrm{l})$ with a series of dilution $(1 / 2,1 / 4,1 / 8,1 / 16,1 / 32$ and 1/64) was delivered to the outside well, and purified BLCAP antigen was delivered to the center well. The plates were incubated for $24 \mathrm{~h}$ and the precipitin reaction was determined.

Patient tissue specimens. A total of 60 cervical specimens consisting of 30 age-matched carcinoma tissues and 30 normal cervical tissues were collected from the Pathology Department at Zhong Nan Hospital (Wuhan University, China) between 2005 and 2006. The patients were evaluated based on the AJCC TNM classification system (10) as: stage I-II ( $n=16)$, stage III-IV $(\mathrm{n}=14) ; 13$ patients had lymphatic metastasis; well-differentiated tumors $(n=16)$ and moderatedly/poorly differentiated tumors $(\mathrm{n}=14)$; squamous cell (SCC) tumors $(n=26)$ and adenosquamous $(A C)$ tumors $(n=4)$. The average age of the patients was 43.3 years ranging from 33 to 63 . None of the patients received radiochemotherapy prior to surgery.

Immunohistochemistry. Sections (5- $\mu$ m thick) from paraffinembedded blocks were mounted on slides. These sections were deparaffinized and then rehydrated with gradient alcohols. Heat-induced antigen retrieval was performed in citrate buffer ( $\mathrm{pH}$ 6.0) by heating the slides in a microwave oven (700 $\mathrm{W}$ for $15 \mathrm{~min}$ ) and then processed for immunohistochemistry. In brief, the tissue sections were washed three times with phosphate-buffered saline (PBS) and incubated with normal horse serum for $30 \mathrm{~min}$ at RT to block non-specific binding. Endogenous peroxidase activity was quenched by incubating sections in $3 \% \mathrm{H}_{2} \mathrm{O}_{2}$ in PBS for 20 min. Sections were then incubated with anti-BLCAP rabbit polyclonal antibody for $60 \mathrm{~min}$ at RT and washed with PBS-T containing $0.05 \%$ Tween-20 (3 x 5 min) before incubating with horseradish peroxidase-labeled secondary antibody for $30 \mathrm{~min}$. Slides were washed again ( 3 x 5 min) with PBS-T. The reaction color was developed by incubating sections with 3,3'-diaminobenzidine reagent (DAB) as per the manufacturer's instructions. The slides were washed with water, counterstained with hematoxylin, dehydrated, mounted and examined under light microscopy. A normal murine $\mathrm{IgG}$ was used for the negative control.

Evaluation of immunohistochemistry (IHC) and statistical analysis. IHC was performed as described previously. Immunostaining was graded as negative (no cells stained), weak $(<10 \%$ cells stained), moderate $(11-50 \%$ cells stained) and strong $(>51 \%)$. For statistical analysis, the staining results were classified into two groups: group I was the 'negative group' (no staining); and group II the 'positive group' (weak intensity, moderate intensity and strong intensity). Statistical analysis for group differences was performed with the $\chi^{2}$ test. For all statistical tests, a P-value $<0.05$ was considered statistically significant.

\section{Results}

Construction of expression plasmid pET-32a-BLCAP. The $264 \mathrm{bp}$ fragment of the BLCAP gene was amplified by PCR. 


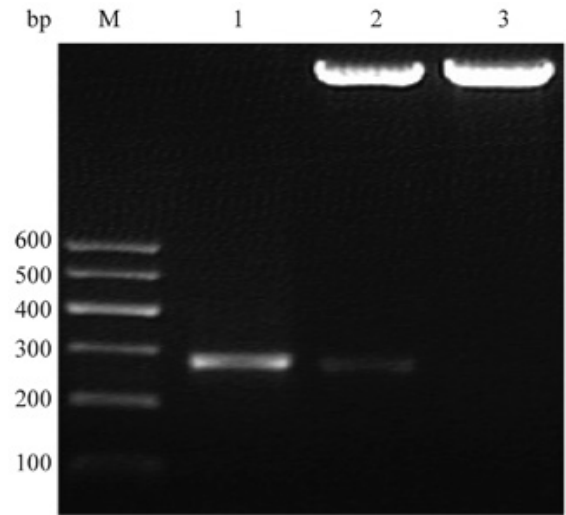

Figure 1. Identification of the recombinant pET-32a-BLCAP by enzymatic digestion. Lane 1, PCR product of the BLCAP gene; lane 2, pET-32a-BLCAP/ EcoRI + HindIII; lane 3, pET-32a EcoRI + HindIII.

a

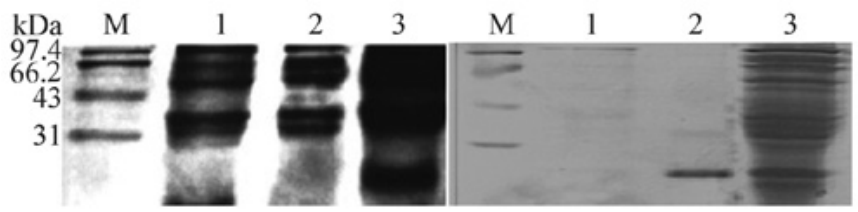

Figure 2. Proteins were separated on $12 \%$ acrylamide gel and stained with Coomassie blue. (a) Expression of the recombinant protein. Lane M, molecular mass markers; lane 1, cells with pET-32a after IPTG induction for $6 \mathrm{~h}$ lane 2, cells with pET-32a-BLCAP before IPTG induction; lane 3, cells with pET-32a-BLCAP after IPTG induction for $6 \mathrm{~h}$. (b) Purification of the recombinant protein. Proteins were separated on $12 \%$ acrylamide gel and stained with Coomassie blue. Lane M, molecular mass markers; lane 1, purified proteins of cells with pET-32a after IPTG induction (as a positive control); lane 2, purified recombinant proteins; lane 3, cells with pET-32 a-BLCAP after IPTG induction. The square frame indicates the BLCAP bands.

The product was cloned into pET-32a and confirmed by PCR and restriction digestion (Fig. 1). DNA sequencing revealed that DNA was the reported sequence. The recombinant plasmid DNA contained the BLCAP gene in-frame with $\mathrm{N}$-terminal Trx-tag and His-tag encoding the $28 \mathrm{kDa}, 762$-amino acid fusion protein.

Expression, purification and analysis of the protein. After induction with IPTG, E. coli Rosetta transformed with pET32a-BLCAP produced a $28-\mathrm{kDa}$ protein shown in Fig. 2a. The size of the protein matched its theoretical molecular weight. To determine the optimal induction period, we used different IPTG concentrations $(0.1,0.2,0.4,0.6,0.8$ and $1.0 \mathrm{mM})$, different temperatures $\left(20,25,30\right.$ and $\left.37^{\circ} \mathrm{C}\right)$, and varied induction times (1, 2, 3, 4, 5, 6, 8 and $12 \mathrm{~h})$. The results showed that the yield was increased with an incubation time and reach a plateau after $6 \mathrm{~h}$. The final concentration of IPTG used in this experiment was $1 \mathrm{mmol} / \mathrm{l}$, and the bacteria were cultivated at $37^{\circ} \mathrm{C}$ for $6 \mathrm{~h}$. Ni-NTA affinity chromatography was applied for purification of the BLCAP fusion protein. The target BLCAP appeared as a single band on SDS-PAGE (Fig. 2b, lane 2), which is in agreement with the molecular weight reported. a

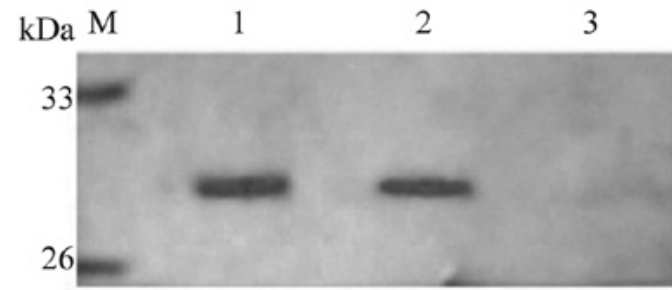

b

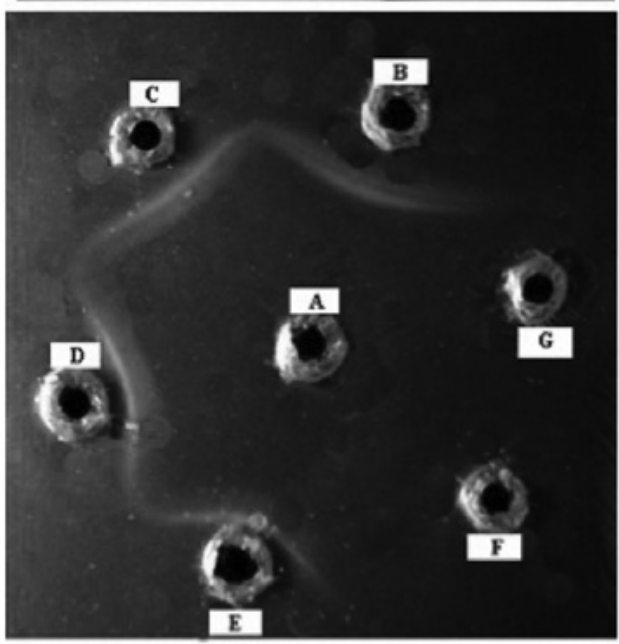

Figure 3. (a) Western blot analysis of recombinant protein using antiserum. Lanes 1 and 2, antiserum; lane 3, pre-immune serum. (b) Determination of antibody titer by agar gel precipitin test, A, antigen, B-G, diluted antiserum, $1 / 2,1 / 4,1 / 8,1 / 16,1 / 32$ and $1 / 64$, respectively.

Western blotting and titer determination. A homogenized preparation containing a single protein for BLCAP could be confidently used for immunizing the New Zealand White rabbit. The antiserum was collected 15 weeks after the initial injection. Three addition booster injections were given and the antiserum was again collected after each booster. A 1:8,000 dilution of the 28-kDa His-tagged BLCAP protein was detected in western blot analysis using IPTG-induced E. coli Rosetta cell extract. There was no immune-reactivity observed in un-induced cells (Fig. 3a). Agar gel precipitin test demonstrated that distinct bands were observed between the antigen and antiserum wells, as a result of the antigen migrating through the agar matrix toward and interacting with antiserum (Fig. 3b).

Tissue immunohistochemistry. The purified polyclonal antibody was used to detect BLCAP protein levels in cervical tissues by immunohistochemistry. This positive expression of BLCAP protein was detected in 27 of $30(90 \%)$ normal cervical tissues with 15 of the 27 (55\%) having moderate to strong cytoplasmic staining. However, in cervical carcinoma tissues 16 of 30 (53.33\%) expressed BLCAP protein with 15 of the $16(93.75 \%)$ having weak cytoplasmic staining (Table I). The expression level of BLCAP in cervical carcinoma was significantly lower than that in the corresponding normal tissues $(\mathrm{P}<0.05)$ (Fig. 4). Moreover, the expression level of BLCAP in cervical carcinoma was significantly associated with clinical degree and tumor differentiation of cervical carcinoma $(\mathrm{P}<0.05)$. No significant difference was observed between SCC and AC (Table II). 
Table I. Immunohistochemical analysis of BLCAP expression in cervical tissues.

\begin{tabular}{lcrrrrr}
\hline & & \multicolumn{3}{c}{ BLCAP expression } & \\
\cline { 3 - 5 } & Total & - & + & ++ & +++ & Positive rate (\%) \\
\hline Normal cervical tissues & 30 & 3 & 8 & $15^{\mathrm{a}}$ & 4 & $90.00^{\mathrm{a}}$ \\
Cervical carcinoma tissues & 30 & 14 & 15 & 1 & 0 & 53.33 \\
\hline
\end{tabular}

${ }^{\mathrm{a}} \mathrm{P}<0.05$.
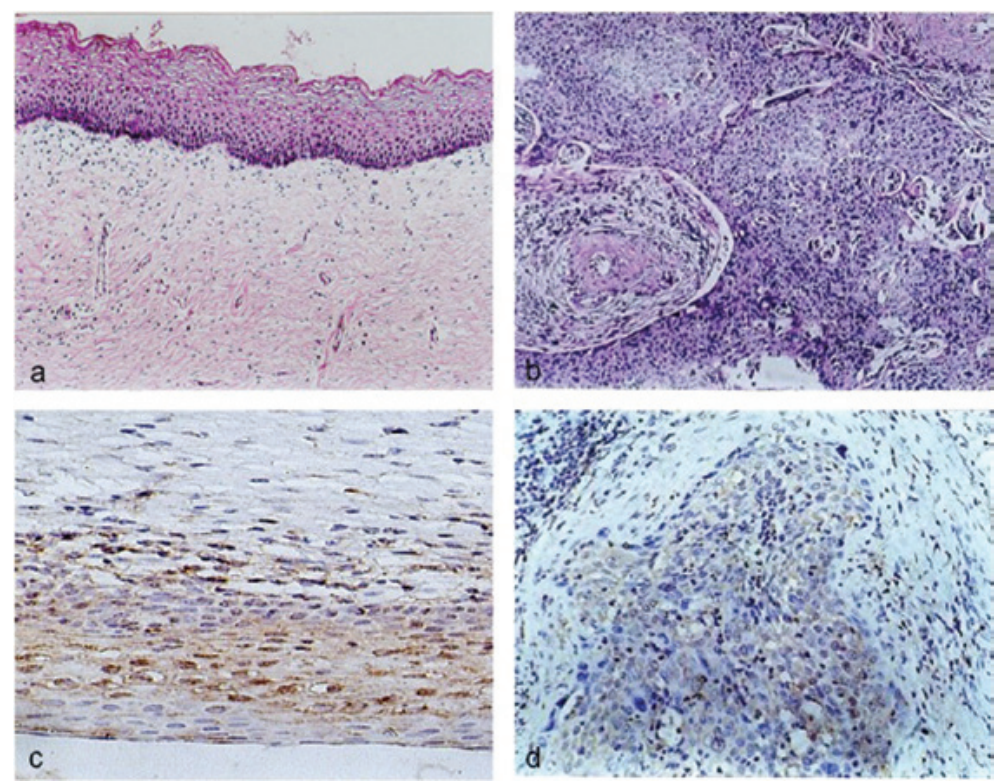

Figure 4. Immunohistochemical staining of BLCAP in normal cervical and cervical carcinomal tissues. (a) H\&E staining in normal cervical tissues (magnification, x100), (b) H\&E staining in cervical carcinoma tissues (magnification, x100), (c) positive expression of BLCAP in normal cervical tissues (magnification, $\mathrm{x} 200$ ), (d) negative expression of BLCAP in cervical carcinoma tissues (magnification, $\mathrm{x} 200$ ).

Table II. Relationship between the expression of BLCAP and the clinical and histopathological features of cervical carcinoma.

\begin{tabular}{lrcr}
\hline & \multicolumn{2}{c}{ BLCAP expression } & \\
$\begin{array}{l}\text { Clinical pathological } \\
\text { characteristic }\end{array}$ & $\mathrm{n}$ & $\mathrm{n}(\%)$ & P-value \\
\hline Histological type & & & \\
$\quad$ SCC & 26 & $14(81.85)$ & $>0.05$ \\
AC & 4 & $2(50)$ & \\
Stage & & & \\
I-II & 16 & $10(62.5)$ & $<0.05$ \\
III-IV & 14 & $6(42.86)$ & \\
Degree of differentiation & & & \\
High & 10 & $7(70)$ & $<0.05$ \\
Moderate/low & 20 & $9(45)$ & \\
Lymphatic metastasis & & & \\
Non-LM & 17 & $12(70.59)$ & $<0.05$ \\
LM & 13 & $4(30.77)$ & \\
Total & 30 & $16(53.55)$ & \\
\hline
\end{tabular}

SCC, squamous cell carcinoma; AC, adenocarcinoma.

\section{Discussion}

The pET-32(a) vector which contains the thioredoxin (Trx) tag was used in this study to successfully produce a BLCAP fusion protein with a sufficient quantum. The Trx tag increases the molecular weight of the fusion protein. Athough this makes the protein more soluble it does not affect its activity $(11,12)$. Western blot analysis revealed the molecular weight of the protein to be approximately $28 \mathrm{kDa}(10 \mathrm{kDa}$ plus the $18 \mathrm{kDa} \mathrm{Tr} x /$ His protein) as predicted. To screen $E$. coli strains for expression, both Rosetta and BL21 were used as host cells to perform prokaryotic expression in the initial stage. The fusion proteins were expressed in the Rosetta but not in BL21. The reason is that BL21 contains rare tRNA codons in the coding sequence of BLCAP, which impedes the expression of eukaryotic protein $(13,14)$. Using Rosetta as the host cell we optimized the culture and induction parameters to get a more soluble fusion protein.

The expressed His-tagged protein was purified by $\mathrm{Ni}^{2+}$ affinity chromatography column and monitored by western blotting with an anti-hexahistidine tag antibody. The purified recombinant proteins were found to be immunogenic in rabbits and produced polyclonal antibodies. Western blot analysis showed that this antibody was highly sensitive and specific. 
The expression rate of BLCAP in cervical carcinoma tissues was significantly lower than that in the normal tissues $(\mathrm{P}<0.05)$. This suggests that decreased expression of BLCAP may be directly related to the development of cervical carcinoma. Meanwhile, the expression of BLCAP was related to clinical stage and cell differentiation, which indicates that the loss of BLCAP may be involved in tumor invasion and metastasis.

In conclusion, a high titer, highly specific BLCAP polyclonal antibody was produced. The differential expression of BLCAP at the protein level was consistent with that of mRNA levels. BLCAP may be a cervical carcinoma-related suppressor gene, which is the foundation for studying the function of BLCAP in clinical applications as a tumor marker.

\section{Acknowledgements}

This study was supported by the National Natural Science Foundation of China (nos. 81072123, 30571955 and 81102024).

\section{References}

1. Szoke K, Sapy T, Krasznai Z, Hernadi Z, Szladek G, Veress G, Dillner J, Gergely L and Konya J: Moderate variation of the oncogenic potential among high-risk human papillomavirus types in gynecologic patients with cervical abnormalities. J Med Virol 71: 585-592, 2003.

2. Bulten J, Melchers WJ, Kooy-Smits MM, de Wilde PC, Poddighe PJ, Robben JC, Macville MV, Massuger LF, Bakkers JM and Hanselaar AG: Numerical aberrations of chromosome 1 in cervical intraepithelial neoplasia are strongly associated with infection with high-risk human papillomavirus types. J Pathol 198: 300-309, 2002.

3. Sarasin A: An overview of the mechanisms of mutagenesis and carcinogenesis. Mutat Res 544: 99-106, 2003.
4. Yokota J: Tumor progression and metastasis. Carcinogenesis 21: 497-503, 2000

5. Moreira JM, Ohlsson G, Gromov P, Simon R, Sauter G, Celis JE and Gromova I: Bladder cancer-associated protein, a potential prognostic biomarker in human bladder cancer. Mol Cell Proteomics 9: 161-177, 2010.

6. Gromova I, Gromov P and Celis JE: Bc10: a novel human bladder cancer-associated protein with a conserved genomic structure downregulated in invasive cancer. Int J Cancer 98: 539-546, 2002.

7. Zuo Z, Zhao M, Liu J, Gao G and Wu X: Functional analysis of bladder cancer-related protein gene: a putative cervical cancer tumor suppressor gene in cervical carcinoma. Tumour Biol 27: 221-226, 2006

8. Zuo ZH, Zhao M, Liu J, Wei Y and Wu XX: Inhibitory effect of bladder cancer related protein gene on hela cell proliferation. $\mathrm{Ai}$ Zheng 25: 811-817, 2006 (In Chinese).

9. Holt PS, Stone HD, Gast RK and Greene CR: Application of the agar gel precipitin test to detect antibodies to Salmonella enterica serovar enteritidis in serum and egg yolks from infected hens. Poult Sci 79: 1246-1250, 2000

10. Stephen B and Edge DRB (eds): AJCC Cancer Staging Manual. 7th edition, Springer, 2009.

11. De Wilde G, Mertens N, Boone E, De Vreese B, Van Beeumen J, Fiers W and Haegeman G: Expression in Escherichia coli of the death domain of the human p55 tumor necrosis factor receptor. Protein Expr Purif 23: 226-232, 2001.

12. Lobel LI, Pollak S, Klein J and Lustbader JW: High-level bacterial expression of a natively folded, soluble extracellular domain fusion protein of the human luteinizing hormone/chorionic gonadotropin receptor in the cytoplasm of escherichia coli. Endocrine 14: 205-212, 2001

13. Wakagi T, Oshima T, Imamura $\mathrm{H}$ and Matsuzawa $\mathrm{H}$ : Cloning of the gene for inorganic pyrophosphatase from a thermoacidophilic archaeon, sulfolobus sp. Strain 7, and overproduction of the enzyme by coexpression of tRNA for arginine rare codon. Biosci Biotechnol Biochem 62: 2408-2414, 1998.

14. Spanjaard RA, Chen K, Walker JR and van Duin J: Frameshift suppression at tandem AGA and AGG codons by cloned tRNA genes: assigning a codon to $\arg \mathrm{U}$ tRNA and T4 tRNA (Arg). Nucleic Acids Res 18: 5031-5036, 1990. 